\title{
SMOOTHNESS AND STRONGLY PSEUDOCONVEXITY OF $p$-WEIL-PETERSSON METRIC
}

\author{
Masahiro Yanagishita \\ Yamaguchi University, Graduate School of Sciences and \\ Technology for Innovation 2-16-1, Department of Applied Science \\ Tokiwadai, Ube-shi, Yamaguchi, 755-8611, Japan; myngsht@yamaguchi-u.ac.jp
}

\begin{abstract}
This paper deals with the smoothness and strongly pseudoconvexity of $p$-WeilPetersson metric. This metric is a complex Finsler metric on the $p$-integrable Teichmüller space of a Riemann surface satisfying Lehner's condition, which is an extended concept of the Weil-Petersson metric on the square integrable Teichmüller space.
\end{abstract}

\section{Introduction}

The Teichmüller space of a Riemann surface of analytically finite type has a complex structure modeled on the complex Hilbert space consisting of harmonic Beltrami differentials on the surface equipped with hyperbolic $L^{2}$-norm. The WeilPetersson metric is an Hermitian metric induced by this Hilbert manifold structure and is studied in many fields. In the complex analysis, Ahlfors $[2,3]$ proved that the Weil-Petersson metric is a Kähler metric and has the negative holomorphic sectional curvature, negative Ricci curvature and negative scalar curvature. In the hyperbolic geometry, Wolpert $[17,18]$ gave the several relations between the Weil-Petersson metric and the Fenchel-Nielsen coordinate.

In general, that Hilbert manifold structure cannot be introduced to the Teichmüller space of a Riemann surface of analytically infinite type (cf. [9]). Takhtajan and Teo [15] realized this structure as a distribution on the universal Teichmüller space. Cui [5] accomplished the same result on the subset of the universal Teichmüller space independently of Takhtajan and Teo. Hui [6] and Tang [16] extended the argument of Cui to the subset modeled on $p$-integrable Beltrami differentials for $p \geq 2$, which we call the $p$-integrable Teichmüller space. Later, Radnell, Schippers and Staubach $[11,12,13]$ composed a Hilbert manifold structure on a certain refined Teichmüller space of a bordered Riemann surface, which is refered to as the WP-class Teichmüller space.

In $[5,15]$, the Weil-Petersson metric was studied for each Hilbert manifold structure. In particular, it was shown that this metric is negatively curved (cf. [15]) and complete (cf. [5]). Recently, Matsuzaki [8] researched some properties of the $p$-WeilPetersson metric on the $p$-integrable Teichmüller space of the unit disk for $p \geq 2$. This metric is a certain extended concept of the Weil-Petersson metric on the square integrable Teichmüller space. In fact, it was proved in [8] that the metric is complete and continuous.

Based on their results, the author [19] introduced some complex analytic structure on the $p$-integrable Teichmüller space of a Riemann surface with Lehner's condition

https://doi.org/10.5186/aasfm.2019.4413

2010 Mathematics Subject Classification: Primary 30F60; Secondary 32G15, 30C60.

Key words: Teichmüller space, $p$-integrable Teichmüller space, Weil-Petersson metric, complex Finsler metric. 
for $p \geq 2$. In particular, this structure is modeled on $p$-integrable holomorphic quadratic differentials, or equivalently on $p$-integrable harmonic Beltrami differentials. We will remark the latter case in Section 2. Here, a Riemann surface satisfies Lehner's condition if the infimum of the length of all simple closed geodesics on the surface is positive.

It is well-known that the metric structure and analytic structure of the Teichmüller space can be inherited from those of the universal Teichmüller space in many cases. In fact, for every hyperbolic Riemann surface $R$, there exists a canonical embedding of the Teichmüller space of $R$ into the universal one, whose image becomes a submanifold. Moreover, the embedding becomes a contraction mapping with respect to their Teichmüller distances. We emphasize that this result does not follow in the $p$-integrable Teichmüller space because the hyperbolic $L^{p}$-norm is affected by the figure of Riemann surfaces better than $L^{\infty}$-norm (see Proposition 2.2). Therefore, we have to study the $p$-integrable Teichmüller space without using the results of Cui, Takhtajan and Teo as they are.

In the recent paper [20], the author proved that the Kählerity and negativity of the Weil-Petersson metric on the square integrable Teichmüller space, which was proved originally for the Teichmüller space of a compact Riemann surface (cf. [2]). We expect that this result is generalized to the $p$-integrable case and that other results given by Ahlfors and Wolpert would also hold in our case, which is our motivation.

This paper corresponds to the first step to study the metric structure of the $p$ integrable Teichmüller space. Our aim is to consider smoothness and strongly pseudoconvexity of the $p$-Weil-Petersson metric because these properties are necessary for our research in the future.

\section{Complex structure on $p$-integrable Teichmüller space}

In this section, we will give the detail of the complex structure on the $p$-integrable Teichmüller space moleded on harmonic Beltrami differentials, which is necessary for the next section. This structure is biholomorphic equivalent to the one obtained from Theorem 4.4 in [19]. Some readers, which are familiar with complex analytic structure of the Teichmüller space, may skip this section because we apply how to construct its structure.

First, we define the $p$-integrable Teichmüller space. Let $\Gamma$ be a Fuchsian group acting on the upper half plane $\mathbf{H}=\{z \in \mathbf{C} \mid \operatorname{Im} z>0\}$ and $\mathcal{L}^{\infty}(\mathbf{H}, \Gamma)$ be the Banach space of Beltrami differentials on $\mathbf{H}$ for $\Gamma$ and finite $L^{\infty}$-norm

$$
\|\mu\|_{\infty}=\operatorname{ess}_{z \in N} \sup |\mu(z)|
$$

where $N$ is a fundamental region on $\mathbf{H}$ for $\Gamma$. Here, a complex valued measurable function $\mu$ on $\mathbf{H}$ is a Beltrami differential for $\Gamma$ if $(\mu \circ \gamma) \overline{\gamma^{\prime}} / \gamma^{\prime}=\mu$ for every $\gamma \in \Gamma$. Let $\operatorname{Bel}(\mathbf{H}, \Gamma)$ be the open unit ball of $\mathcal{L}^{\infty}(\mathbf{H}, \Gamma)$. Each element in $\operatorname{Bel}(\mathbf{H}, \Gamma)$ is called a Beltrami coefficient on $\mathbf{H}$ for $\Gamma$. For $\mu \in \operatorname{Bel}(\mathbf{H}, \Gamma)$, let $f^{\mu}$ be the quasiconformal self-mapping of $\mathbf{H}$ satisfying the Beltrami equation $\bar{\partial} f=\mu \partial f$ and normalized by fixing $0,1, \infty$. Two Beltrami coefficients $\mu, \nu \in \operatorname{Bel}(\mathbf{H}, \Gamma)$ are Teichmüller equivalent if $\left.f^{\mu}\right|_{\mathbf{R}}=\left.f^{\nu}\right|_{\mathbf{R}}$. Then the Teichmüller space $T(\Gamma)$ of $\Gamma$ is defined as the quotient space of $\operatorname{Bel}(\mathbf{H}, \Gamma)$ by the Tei chmüller equivalence relation. Each element of the Teichmüller space is called a Teichmüller equivalence class. Let $[\mu]$ be the Teichmüller equivalence class represented by $\mu \in \operatorname{Bel}(\mathbf{H}, \Gamma)$. In particular, the Teichmüller equivalence class represented by the zero function on $\mathbf{H}$ is called the base point, denoted by 0 . For a linear map $A$ on $\mathcal{L}^{\infty}(\mathbf{H}, \Gamma)$, we usually use the representation 
$A[\mu]$ as the image of $\mu \in \mathcal{L}^{\infty}(\mathbf{H}, \Gamma)$. Hereafter, the reader should be careful so that he/she does not confuse how to use the bracket "[ ]".

For $p \geq 1$, let $\mathcal{L}^{p}(\mathbf{H}, \Gamma)$ be the Banach space of Beltrami differentials for $\Gamma$ with finite hyperbolic $L^{p}$-norm

$$
\|\mu\|_{p}=\left(\int_{N}|\mu(z)|^{p} \rho_{\mathbf{H}}(z)^{2} d \sigma(z)\right)^{\frac{1}{p}},
$$

where $\rho_{\mathbf{H}}(z)=(2 \operatorname{Im} z)^{-1}$ is the Poincaré metric on $\mathbf{H}$ and $d \sigma(z)=d x d y$ for $z=$ $x+i y$. Set $\operatorname{Ael}^{p}(\mathbf{H}, \Gamma)=\operatorname{Bel}(\mathbf{H}, \Gamma) \cap \mathcal{L}^{p}(\mathbf{H}, \Gamma)$. The $p$-integrable Teichmüller space $T^{p}(\Gamma)$ of a Fuchsian group $\Gamma$ is defined by $T^{p}(\Gamma)=\left\{[\mu] \in T(\Gamma) \mid \mu \in \operatorname{Ael}^{p}(\mathbf{H}, \Gamma)\right\}$.

Next, we introduce the complex structure on $T^{p}(\Gamma)$. Let $\mathbf{H}^{*}=\{z \in \mathbf{C} \mid \operatorname{Im} z<0\}$ be the lower half plane. A quadratic differential $\varphi$ on $\mathbf{H}^{*}$ for $\Gamma$ is bounded if the hyperbolic $L^{\infty}$-norm

$$
\|\varphi\|_{\infty}=\sup _{z \in N^{*}}|\varphi(z)| \rho_{\mathbf{H}^{*}}(z)^{-2}
$$

is finite. Here $N^{*}$ is a fundamental region of $\mathbf{H}^{*}$ for $\Gamma$ and $\rho_{\mathbf{H}^{*}}(z)=(-2 \operatorname{Im} z)^{-1}$ is the Poincaré metirc on $\mathbf{H}^{*}$. Similarly, A quadratic differential $\varphi$ on $\mathbf{H}^{*}$ for $\Gamma$ is $p$-integrable if the hyperbolic $L^{p}$-norm

$$
\|\varphi\|_{p}=\left(\int_{N^{*}}|\varphi(z)|^{p} \rho_{\mathbf{H}^{*}}(z)^{2-2 p} d \sigma(z)\right)^{\frac{1}{p}}
$$

is finite. Let $\mathcal{B}\left(\mathbf{H}^{*}, \Gamma\right)$ and $A^{p}\left(\mathbf{H}^{*}, \Gamma\right)$ be the Banach space and the Hilbert space of bounded and square integrable holomorphic quadratic differentials on $\mathbf{H}^{*}$ for $\Gamma$, respectively.

There exists a relation between the hyperbolic $L^{p}$-norm and $L^{\infty}$-norm for Fuchsian groups with Lehner's condition:

Proposition 2.1. [7, 10, 14] A Fuchsian group $\Gamma$ satisfies Lehner's condition if and only if there exist an index $p \geq 1$ and a constant $C_{p}(\Gamma)$ depending only on $\Gamma$ and $p$ such that

$$
\|\varphi\|_{\infty} \leq C_{p}(\Gamma)\|\varphi\|_{p}
$$

for every $\varphi \in A^{p}\left(\mathbf{H}^{*}, \Gamma\right)$. Especially, $A^{p}\left(\mathbf{H}^{*}, \Gamma\right)$ is contained in $\mathcal{B}\left(\mathbf{H}^{*}, \Gamma\right)$.

Let us introduce harmonic Beltrami differentials. Set

$$
\mu[\varphi](z)=\frac{1}{4} \rho_{\mathbf{H}}(z)^{-2} \overline{\varphi(z)}=(\operatorname{Im} z)^{2} \overline{\varphi(z)}
$$

for $\varphi \in A^{p}(\mathbf{H}, \Gamma)$, where $A^{p}(\mathbf{H}, \Gamma)$ is the Hilbert space of $p$-integrable holomorphic quadratic differentials on $\mathbf{H}$ for $\Gamma$. Let $\mathcal{L}^{p, \infty}(\mathbf{H}, \Gamma)=\mathcal{L}^{p}(\mathbf{H}, \Gamma) \cap \mathcal{L}^{\infty}(\mathbf{H}, \Gamma)$. We see that $\mathcal{L}^{p, \infty}(\mathbf{H}, \Gamma)$ forms a Banach space equipped with norm $\|\cdot\|_{p, \infty}=\|\cdot\|_{p}+\|\cdot\|_{\infty}$. Then $\mu[\varphi]$ belongs to $\mathcal{L}^{p, \infty}(\mathbf{H}, \Gamma)$.

For a holomorphic function $f$, the function

$$
S_{f}=\left(\frac{f^{\prime \prime}}{f^{\prime}}\right)^{\prime}-\frac{1}{2}\left(\frac{f^{\prime \prime}}{f^{\prime}}\right)^{2}
$$

is the Schwarzian derivative of $f$. For $\mu \in \operatorname{Bel}(\mathbf{H}, \Gamma)$, we extend $\mu$ to a function on $\mathbf{C}$ by letting 0 on $\mathbf{H}^{*}$. Then $f_{\mu}$ is defined as the quasiconformal mapping on $\hat{\mathbf{C}}$ with the extended Beltrami coefficient $\mu$ that is normalized by $\lim _{z \rightarrow \infty}\left(f_{\mu}(z)-z\right)=0$. Let

$$
\varphi[\mu](z)=-2 \overline{D \Phi(0)[\mu](\bar{z})}=\frac{12}{\pi} \int_{\mathbf{H}} \frac{\overline{\mu(\zeta)}}{\overline{(\bar{\zeta}-z)^{4}}} d \sigma(\zeta)
$$


for $\mu \in \mathcal{L}^{p, \infty}(\mathbf{H}, \Gamma)$ and $z \in \mathbf{H}$. Here $\Phi(\mu)=S_{\left.f_{\mu}\right|_{\mathbf{H}^{*}}}$ is the Bers projection and $D \Phi$ means the Fréchet derivative of $\Phi$. Note that $\Phi$ is a holomorphic map of $\operatorname{Ael}^{p}(\mathbf{H}, \Gamma)$ into $A^{p}\left(\mathbf{H}^{*}, \Gamma\right)$. It follows that

$$
\varphi[\mu]=4 b_{2}\left(\rho_{\mathbf{H}}^{2} \bar{\mu}\right)
$$

for every $\mu \in \mathcal{L}^{p, \infty}(\mathbf{H}, \Gamma)$. Here $b_{2}$ is the Bergman projection. In other words,

$$
b_{2}(\varphi)(z)=\frac{3}{\pi} \int_{\mathbf{H}} \rho_{\mathbf{H}}(\zeta)^{-2} \varphi(\zeta) \overline{K_{\mathbf{H}}(z, \zeta)} d \sigma(z)
$$

for a measurable quadratic differential $\varphi$, where $K_{\mathbf{H}}(z, \zeta)=(\bar{z}-\zeta)^{-4}$ is the Bergman kernel on $\mathbf{H}$. Then $\varphi[\mu]$ is an element of $A^{p}(\mathbf{H}, \Gamma)$. It follows from formula (2.3) and the reproducing formula of the Bergman kernel that for every $\varphi \in A^{p}(\mathbf{H}, \Gamma)$,

$$
\varphi[\mu[\varphi]]=\varphi .
$$

Set

$$
H[\mu](z)=\mu[\varphi[\mu]](z)=-\frac{1}{2} \rho_{\mathbf{H}}(z)^{-2} D \Phi(0)[\mu](\bar{z})
$$

for $\mu \in \mathcal{L}^{p, \infty}(\mathbf{H}, \Gamma)$, which is called the harmonic Beltrami differential for $\mu$ and $z \in \mathbf{H}$. By formula (2.4), we have

$$
H[\mu[\varphi]]=\mu[(\varphi[\mu[\varphi]])]=\mu[\varphi]
$$

for every $\varphi \in A^{p}(\mathbf{H}, \Gamma)$. This fact implies that

$$
H^{2}=H \circ H=H \text {. }
$$

In other words, $H$ is a projection of $\mathcal{L}^{p, \infty}(\mathbf{H}, \Gamma)$. If we set $\operatorname{HB}^{p}(\mathbf{H}, \Gamma)=H\left(\mathcal{L}^{p, \infty}(\mathbf{H}, \Gamma)\right)$, then we have the direct sum decomposition

$$
\mathcal{L}^{p, \infty}(\mathbf{H}, \Gamma)=\operatorname{HB}^{p}(\mathbf{H}, \Gamma) \oplus \operatorname{Ker} H .
$$

Since $H$ is a bounded linear operator, $\operatorname{HB}^{p}(\mathbf{H}, \Gamma)$ is a Banach subspace of $\mathcal{L}^{p, \infty}(\mathbf{H}, \Gamma)$.

Now let us introduce a complex structure on $T^{p}(\Gamma)$.

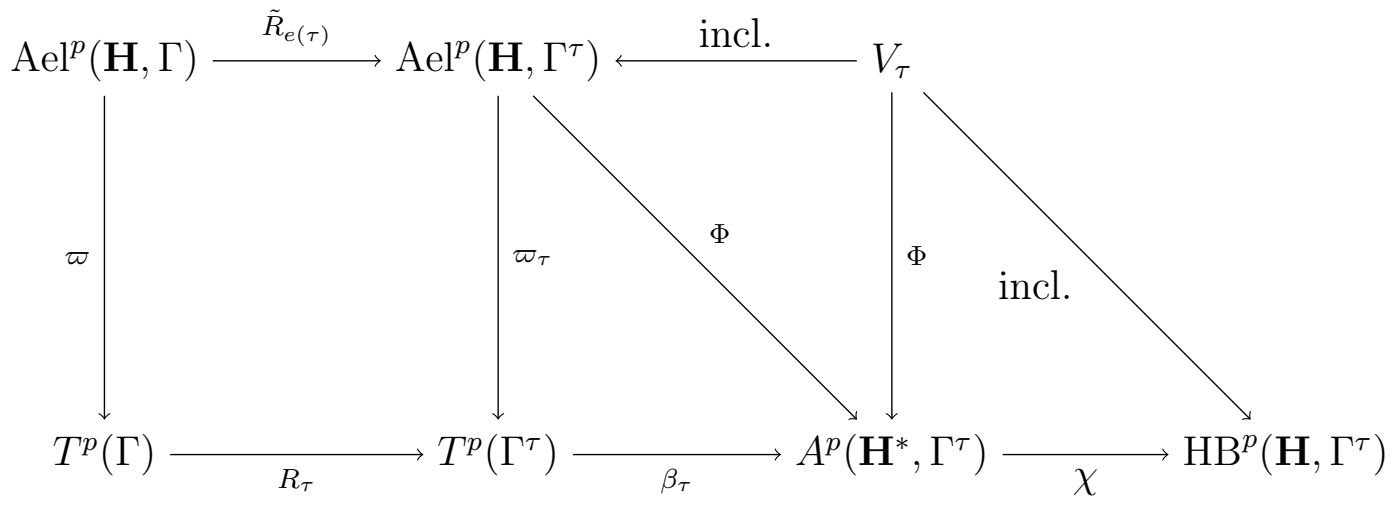

Figure.

For $\tau \in T(\Gamma)$, let $\Gamma^{\tau}=f^{\nu} \Gamma\left(f^{\nu}\right)^{-1}$ be the Fuchsian group deformed by $\tau$ where $\nu \in$ $\tau$. We note that $\Gamma^{\tau}$ is defined independently of the choice of $\nu$. Let $\varpi_{\tau}: \operatorname{Bel}\left(\mathbf{H}, \Gamma^{\tau}\right) \rightarrow$ $T\left(\Gamma^{\tau}\right)$ be the canonical projection and

$$
\beta_{\tau}([\mu])=S_{\left.f_{\mu}\right|_{\mathbf{H}^{*}}}
$$

be the Bers embedding on $T\left(\Gamma^{\tau}\right)$. It is known that $\beta_{\tau}$ is a homeomorphism of $T\left(\Gamma^{\tau}\right)$ into $\mathcal{B}\left(\mathbf{H}^{*}, \Gamma^{\tau}\right)$. Theorem 4.4 in [19] means that $\beta_{\tau}$ is also a homeomorphism of $T^{p}\left(\Gamma^{\tau}\right)$ into $A^{p}\left(\mathbf{H}^{*}, \Gamma^{\tau}\right)$. In the proof, Proposition 2.1 is applied essentially. 
Let $B_{p}(\tau)=\left\{\varphi \in A^{p}\left(\mathbf{H}^{*}, \Gamma^{\tau}\right) \mid\|\varphi\|_{p}<2 / C_{p}\left(\Gamma^{\tau}\right)\right\}$. By inequality (2.1), we have $\|\varphi\|_{\infty}<2$ for every $\varphi \in B_{p}(\tau)$. Then it follows that for every $\varphi \in B_{p}(\tau)$, the Ahlfors-Weill section of $\varphi$

$$
\chi(\varphi)(z)=-\frac{1}{2} \rho_{\mathbf{H}^{*}}(\bar{z})^{-2} \varphi(\bar{z})=\mu[-2 \overline{\varphi(\bar{z})}] \quad(z \in \mathbf{H})
$$

belongs to $\operatorname{HB}^{p}\left(\mathbf{H}, \Gamma^{\tau}\right)$. Indeed, $\chi$ is an isometry of $A^{p}\left(\mathbf{H}^{*}, \Gamma^{\tau}\right)$ onto $\operatorname{HB}^{p}\left(\mathbf{H}, \Gamma^{\tau}\right)$ by formula (2.5).

For $\mu, \nu \in \operatorname{Bel}(\mathbf{H}, \Gamma)$, let $\tilde{R}_{\nu}(\mu)$ be the Beltrami coefficient of $f^{\mu} \circ\left(f^{\nu}\right)^{-1}$ and $R_{[\nu]}([\mu])=\left[\tilde{R}_{\nu}(\mu)\right]$. We call $\tilde{R}_{\nu}$ and $R_{[\nu]}$ the right translation map for $\nu$ and $[\nu]$, respectively. Let $\operatorname{DE}(\tau)$ be the Douady-Earle extension for $\tau$ and $e(\tau)$ be the Beltrami coefficient of $g_{1} \circ \mathrm{DE}(\tau) \circ g_{2}^{-1}$, where $g_{1}, g_{2}$ are the conformal maps of $\mathbf{D}=\{z \in$ $\mathbf{C}|| z \mid<1\}$ onto $\mathbf{H}$ such that $g_{1} \circ \operatorname{DE}(\tau) \circ g_{2}^{-1}$ fixes $0,1, \infty$. By Proposition 5.1 in [19], the right translation map $\tilde{R}_{e(\tau)}\left(\operatorname{resp} . R_{\tau}\right)$ is a homeomorphism of $\operatorname{Ael}^{p}(\mathbf{H}, \Gamma)$ onto $\operatorname{Ael}^{p}\left(\mathbf{H}, \Gamma^{\tau}\right)$ (resp. of $T^{p}(\Gamma)$ onto $\left.T^{p}\left(\Gamma^{\tau}\right)\right)$.

Set $V_{\tau}=\chi\left(B_{p}(\tau)\right) \subset \operatorname{HB}^{p}(\mathbf{H}, \Gamma)$. Then the collection

$$
\left\{U_{\tau}=\varpi \circ \tilde{R}_{e(\tau)}^{-1}\left(V_{\tau}\right) \mid \tau \in T^{p}(\Gamma)\right\}
$$

is an open covering of $T^{p}(\Gamma)$. In fact, $\tau$ belongs to $U_{\tau}$ for every $\tau \in T^{p}(\Gamma)$ and $U_{\tau}$ coincides with the preimage of the open set $V_{\tau}$ under the homeomorphism $\varsigma_{\tau}=$ $\chi \circ \beta_{\tau} \circ R_{\tau}$. Thus we obtain the family

$$
\mathcal{A}=\left\{\left(U_{\tau}, \varsigma_{\tau}\right) \mid \tau \in T^{p}(\Gamma)\right\}
$$

It follows that $\mathcal{A}$ forms an atlas of $T^{p}(\Gamma)$ for every $p \geq 2$ and every Fuchsian group with Lehner's condition. We omit the proof since it is given almost similarly to the proof of Theorem 2.3 in [20] by replacing the letter " 2 " with " $p$ " and by the post-composition of $\lambda_{\tau}=\beta_{\tau} \circ R_{\tau}$ by $\chi$.

Since a chart $\left(U_{\tau}, \varsigma_{\tau}\right)$ is modeled on the Banach space $\operatorname{HB}^{p}\left(\mathbf{H}, \Gamma^{\tau}\right)$ for every $\tau \in T^{p}(\Gamma)$, we obtain the following representation of the holomorphic tangent space of $T^{p}(\Gamma)$ at $\tau$ :

$$
T_{\tau}^{1,0} T^{p}(\Gamma) \simeq \operatorname{HB}^{p}\left(\mathbf{H}, \Gamma^{\tau}\right) .
$$

We end this section by remarking the relation between $p$-integrable $(-1,1)$-differentials for two Fuchsian groups.

Proposition 2.2. Let $\Gamma$ be a Fuchsian group and $\Gamma^{\prime}$ be a subgroup of $\Gamma$. Then the following three conditions are equivalent:

(1) $\left[\Gamma: \Gamma^{\prime}\right]<\infty$;

(2) $\mathcal{L}^{p}(\mathbf{H}, \Gamma)$ is a subspace of $\mathcal{L}^{p}\left(\mathbf{H}, \Gamma^{\prime}\right)$ as a Banach space;

(3) $\left(\mathcal{L}^{p}(\mathbf{H}, \Gamma) \cap \mathcal{L}^{p}\left(\mathbf{H}, \Gamma^{\prime}\right)\right) \backslash\{0\} \neq \emptyset$.

The proof is given similarly to Proposition 2.4 in [20].

For every subgroup $\Gamma^{\prime}$ of $\Gamma$, the Banach space $\mathcal{L}^{\infty}(\mathbf{H}, \Gamma)$ is a subspace of $\mathcal{L}^{\infty}\left(\mathbf{H}, \Gamma^{\prime}\right)$ since their $L^{\infty}$-norms coincide in $\mathcal{L}^{\infty}(\mathbf{H}, \Gamma)$. Comparing this point with the above proposition, we see that the metric and complex structures of $T^{p}(\Gamma)$ does not inherit those of $T^{p}\left(\Gamma^{\prime}\right)$ generally, and we emphasize that our study is not given as a corollary of the results obtained in [2] and [15] for infinitely analytic type Fuchsian groups.

\section{Definition of $p$-Weil-Petersson metric}

In this section, we define the $p$-Weil-Petersson metric on the $p$-integrable Teichmüller space. We first introduce the complex Finsler metric. 
Definition 3.1. Let $M$ be a complex manifold modeled on a Banach space. A real valued function $F$ of the holomorphic tangent bundle $T^{1,0} M$ is a complex Finsler metric on $M$ if the following two conditions are satisfied:

(1) For every $(z, v) \in T^{1,0} M, F(z, v) \geq 0$, and $F(z, v)=0$ if and only if $v=0$;

(2) For every $(z, v) \in T^{1,0} M$ and $\lambda \in \mathbf{C}, F(z, \lambda v)=|\lambda| F(z, v)$.

For example, the Kobayashi metric and Carathéodory metric on a complex manifold $M$ are upper semicontinuous complex Finsler metrics. Other example is the Hermitian metric on $M$, which induces a canonical $C^{\infty}$-smooth complex Finsler metric. Here, a complex Finsler metric on $M$ is $C^{n}$-smooth for $n \in \mathbf{N} \cup\{\infty\}$ if $F$ is $C^{n}$-smooth on $T^{1,0} M \backslash\{0\}$, where 0 means the zero section on the holomorphic tangent bundle $T^{1,0} M$. In fact, if $F$ is $C^{2}$-smooth on $T^{1,0} M$, then $F$ is induced by an Hermitian metric on $M$. Note that the complex Finsler metric in many other papers is defined as the $C^{\infty}$-smooth one in this paper.

Let $z \in M$ and $F$ be a complex Finsler metric on $M$ that $F(z, \cdot)$ is twice differentiable on the holomorphic tangent space $T_{z}^{1,0} M$. Then $F$ is strongly pseudoconvex at $z$ if

$$
\left.\frac{\partial^{2} G}{\partial t \partial \bar{t}}(z, v+t \zeta)\right|_{t=0}>0
$$

for every $v, \zeta \in T_{z}^{1,0} M \backslash\{0\}$, where $G=F^{2}$. If $F$ is strongly pseudoconvex at every $z \in M$, then $F$ is called strongly pseudoconvex on $M$. When $M$ is a finite dimensional manifold, this condition means that the Levi matrix $\left\{G_{\alpha \beta}(z)\right\}$ is positive definite for every $z \in M$. The strong pseudoconvexity allows us to define a Hermitian metric on the vertical bundle of $M$ and to consider its holomorphic flag curvature (see Chapter 2 in [1]).

In order to define the $p$-Weil-Petersson metric, we deal with the coordinate transformation on the tangent bundle $T^{1,0} T^{p}(\Gamma)$. Take $\tau, \tau^{\prime} \in T^{p}(\Gamma)$ with $U_{\tau} \cap U_{\tau^{\prime}} \neq \emptyset$ and $\xi \in U_{\tau} \cap U_{\tau^{\prime}}$. Since every chart of $\mathcal{A}$ is determined uniquely by some point in $T^{p}(\Gamma)$, we may assume $\tau^{\prime}=\xi$. Let us consider the derivative of the coordinate transformation $\varsigma_{\tau \xi}=\varsigma_{\xi} \circ \varsigma_{\tau}^{-1}: \varsigma_{\tau}\left(U_{\tau} \cap U_{\xi}\right) \rightarrow \varsigma_{\xi}\left(U_{\tau} \cap U_{\xi}\right)$. It clearly follows that $\varsigma_{\tau \xi}\left(\varsigma_{\tau}(\xi)\right)=0$. Since $\nu_{\tau}(\xi)=\tilde{R}_{e(\tau)}^{-1}\left(\varsigma_{\tau}(\xi)\right)$ belongs to the Teichmüller equivalence class $\xi, \tilde{R}_{\nu_{\tau}(\xi)}$ is a lift of $R_{\xi}$. In addition, $\tilde{R}_{\nu_{\tau}(\xi)}=\tilde{R}_{\varsigma_{\tau}(\xi)} \circ \tilde{R}_{e(\tau)}$ by the definition of righ t translation maps. Then we have

$$
\begin{aligned}
\varsigma_{\tau \xi} & =\varsigma_{\tau} \circ \varsigma_{\xi}^{-1}=\left(\chi \circ \beta_{\xi} \circ R_{\xi}\right) \circ\left(\varpi \circ \tilde{R}_{e(\tau)}^{-1}\right) \\
& =\chi \circ \beta_{\xi} \circ\left(\varpi_{\xi} \circ \tilde{R}_{\nu_{\tau}(\xi)}\right) \circ \tilde{R}_{e(\tau)}^{-1}=\chi \circ \Phi \circ \tilde{R}_{\varsigma_{\tau}(\xi)} .
\end{aligned}
$$

This implies that

$$
\begin{aligned}
D \varsigma_{\tau \xi}\left(\varsigma_{\tau}(\xi)\right) & =D\left(\chi \circ \Phi \circ \tilde{R}_{\varsigma_{\tau}(\xi)}\right)\left(\varsigma_{\tau}(\xi)\right) \\
& =\chi \circ D \Phi(0) \circ D \tilde{R}_{\varsigma_{\tau}(\xi)}\left(\varsigma_{\tau}(\xi)\right)=H \circ L^{\varsigma_{\tau}(\xi)},
\end{aligned}
$$

where $L^{\varsigma_{\tau}(\xi)}=D \tilde{R}_{\varsigma_{\tau}(\xi)}\left(\varsigma_{\tau}(\xi)\right)$ is a bounded linear isomorphism of $\mathcal{L}^{p, \infty}(\mathbf{H}, \Gamma)$ onto $\mathcal{L}^{p, \infty}\left(\mathbf{H}, \Gamma^{\xi}\right)$.

Therefore, in an open neighborhood $U_{\tau} \subset T^{p}(\Gamma)$, the $p$-Weil-Petersson metric on $U_{\tau}$ is defined as

$$
h_{\mathrm{WP}}^{p}(\xi, \mu)=\left\|H \circ L^{\varsigma_{\tau}(\xi)}[\mu]\right\|_{p, \tau}
$$

for $\xi \in U_{\tau}$ and $\mu \in \operatorname{HB}^{p}\left(\mathbf{H}, \Gamma^{\tau}\right) \simeq T_{\tau}^{1,0} T^{p}(\Gamma)$. Here, $\|\cdot\|_{p, \tau}$ is the hyperbolic $L^{p}$-norm of $\mathcal{L}^{p}\left(\mathbf{H}, \Gamma^{\tau}\right)$. From the argument above, the holomorphic tangent vector $(\xi, \mu) \in U_{\tau} \times$ 
$\operatorname{HB}^{p}\left(\mathbf{H}, \Gamma^{\tau}\right)$ is identified with $\left(\xi, H \circ L^{\varsigma_{\tau}}(\xi)[\mu]\right) \in U_{\xi} \times \operatorname{HB}^{p}\left(\mathbf{H}, \Gamma^{\xi}\right)$. This observation implies that $h_{\mathrm{WP}}^{p}$ is invariant under coordinate transformations, that is, $h_{\mathrm{WP}}^{p}$ becomes a complex valued function on the holomorphic tangent bundle $T^{1,0} T^{p}(\Gamma)$. Moreover, since $\|\cdot\|_{p, \tau}$ is a norm, it clearly follows that $h_{\mathrm{WP}}^{p}$ becomes a complex Finsler metric on $T^{p}(\Gamma)$.

Remark. In [8], the $p$-Weil-Petersson metric is defined for the Banach manifold structure modeled on the Banach space $A^{p}\left(\mathbf{D}^{*}\right)$ of $p$-integrable holomorphic quadratic differential on $\mathbf{D}^{*}=\{z \in \mathbf{C}|| z \mid>1\}$. Since $\mathbf{D}^{*}$ is conformally equivalent to the lower half plane $\mathbf{H}^{*}$ and $\operatorname{HB}^{p}(\mathbf{H}, 1)$ is isometrically isomorphic to $A^{p}\left(\mathbf{H}^{*}\right)$, our definition is essentially equivalent to Matsuzaki's one when $\Gamma=1$. Here, $1=\left\{i d_{\mathbf{H}}\right\}$ means the trivial group.

\section{Some lemmas}

This section is devoted to some lemmas for the main theorem. Let $K(z, w)=$ $(z-w)^{-2}$ for $z, w \in \mathbf{C}$. For $\mu \in \operatorname{Bel}(\mathbf{H}, 1)$, set

$$
K_{\mu}(z, \bar{w})=K\left(f^{\mu}(z), \overline{f^{\mu}(w)}\right) \partial f^{\mu}(z) \overline{\partial f^{\mu}(w)}=\frac{\partial f^{\mu}(z) \overline{\partial f^{\mu}(w)}}{\left(f^{\mu}(z)-\overline{f^{\mu}(w)}\right)^{2}}
$$

and $F_{z}(\mu)=-K_{\mu}(z, \bar{z})$. The operator $F_{z}(\mu)$ means the deformation of the Poincaré metric by $\mu$ since $F_{z}(0)=-K(z, \bar{z})=\rho_{\mathbf{H}}(z)^{2}$. There exists some estimation for $F_{z}(\mu)$ around $\mu=0$ as follows:

Lemma 4.1. [15, Lemma 2.5] For every $\varepsilon>0$, there exists a constant $0<\delta<1$ such that for every $\mu \in \operatorname{HB}^{\infty}(\mathbf{H}, 1)$ with $\|\mu\|_{\infty}<\delta$ and every $z \in \mathbf{H}$,

$$
\left|F_{z}(\mu)-\rho_{\mathbf{H}}(z)^{2}\right|<\varepsilon \rho_{\mathbf{H}}(z)^{2} .
$$

We note that this lemma is actually given for the unit disk $\mathbf{D}$ instead of $\mathbf{H}$ in [15]. Since $\mathbf{D}$ is conformally equivalent to $\mathbf{H}$, this lemma clearly follows.

For a (Fréchet) differentiable complex valued functional $f$ on a domain $U$ of a complex Banach space $B$, let us define

$$
\begin{aligned}
D^{\prime} f(z)[v] & =\frac{1}{2}(D f(z)[v]-i D f(z)[i v]), \\
D^{\prime \prime} f(z)[v] & =\frac{1}{2}(D f(z)[v]+i D f(z)[i v])
\end{aligned}
$$

for $(z, v) \in U \times B$. Then $D^{\prime} f(z)$ is a bounded complex linear functional and $D^{\prime \prime} f(z)$ is a bounded complex anti-linear functional. If $D^{\prime \prime} f(z) \equiv 0$, then $f$ is holomorphic at $z$. Moreover, it follows that $D^{\prime} f(z)=D f(z)$.

The next lemma is an analogy of Lemma 4.1 for the first variation of $F_{z}$.

Lemma 4.2. For every $\varepsilon>0$, there exists a constant $0<\delta<1$ such that for every $\mu \in \operatorname{HB}^{\infty}(\mathbf{H}, 1)$ with $\|\mu\|_{\infty}<\delta, \kappa \in \operatorname{HB}^{\infty}(\mathbf{H}, 1)$ and $z \in \mathbf{H}$,

$$
\left|D^{\prime} F_{z}(\mu)[\kappa]\right|<\varepsilon\|\kappa\|_{\infty} \rho_{\mathbf{H}}(z)^{2} .
$$

Proof. Fix $z_{0} \in \mathbf{H}$ arbitrarily. Let

$$
A(w)=\frac{w-\operatorname{Re} f^{\mu}\left(z_{0}\right)}{\operatorname{Im} f^{\mu}\left(z_{0}\right)}, \quad B(z)=\left(\operatorname{Im} z_{0}\right) z+\operatorname{Re} z_{0} .
$$


Then $A, B$ are Möbius transformations preserving $\mathbf{H}$. Set $g^{\mu}=A \circ f^{\mu} \circ B$. By simple computation, $g^{\mu}$ fixes $i$ and satisfies

$$
\left|\partial g^{\mu}(i)\right|^{2}=\rho_{\mathbf{H}}\left(z_{0}\right)^{-2} F_{z_{0}}(\mu) .
$$

It clearly follows that the function $(\mu, z) \mapsto f^{\mu}(z)$ is $C^{\infty}$-smooth on $U \times \mathbf{H}$, where $U$ is a sufficiently small neighborhood at 0 in $\operatorname{HB}^{\infty}(\mathbf{H}, 1)$ (see Theorem 11 in [4]). This implies that the function $G_{z_{0}}: \mu \mapsto\left|\partial g^{\mu}(i)\right|^{2}$ is also $C^{\infty}$-smooth in $U$. In particular, its $z$-derivative $D^{\prime} G_{z_{0}}=\rho_{\mathbf{H}}\left(z_{0}\right)^{-2} D^{\prime} F_{z_{0}}$, which is a map of $\mathrm{U}$ into the Banach space of bounded complex linear functionals on $\operatorname{HB}^{\infty}(\mathbf{H}, 1)$, is continuous at $\mu=0$. Hence for every $\varepsilon>0$ there exists a positive constant $\delta>0$ such that

$$
\left\|D^{\prime} G_{z_{0}}(\mu)-D^{\prime} G_{z_{0}}(0)\right\|<\varepsilon
$$

for every $\mu \in \operatorname{HB}^{\infty}(\mathbf{H}, 1)$ with $\|\mu\|_{\infty}<\delta$, that is,

$$
\left|D^{\prime} G_{z_{0}}(\mu)[\kappa]-D^{\prime} G_{z_{0}}(0)[\kappa]\right|<\varepsilon\|\kappa\|_{\infty} .
$$

By Lemma 2 in [3], we have

$$
D^{\prime} F_{z_{0}}(0)[\nu]=-\left.\frac{\partial}{\partial t} K_{t \nu}\left(z_{0}, \bar{z}_{0}\right)\right|_{t=0}=0
$$

for every $\nu \in \mathrm{HB}^{\infty}(\mathbf{H}, 1)$. This result and formula (4.1) imply that

$$
\rho_{\mathbf{H}}\left(z_{0}\right)^{-2}\left|D^{\prime} F_{z_{0}}(\mu)[\kappa]\right|<\varepsilon\|\kappa\|_{\infty} .
$$

Since $z_{0}$ is taken arbitrarily, the lemma holds.

Let us denote by $B_{e}(z, r)$ and $B_{h}(z, r)$ the Euclidean and the hyperbolic open ball centered at $z$ of radius $r$, respectively. The following lemma is a generalization of Lemma 3.2 in [20] for $p \geq 2$.

Lemma 4.3. For $p \geq 2$ and $r>e$, let $u(r)=\log \log r$ and $\mathbf{H}(r)=\{z \in \mathbf{H} \mid$ $|z|>r\}$. Then

as $r \rightarrow \infty$.

$$
E(r)=\int_{B_{h}(i, u(r))} \rho_{\mathbf{H}}(z)^{2-p}\left(\int_{\mathbf{H}(r)}|K(\bar{z}, \zeta)|^{2} d \sigma(\zeta)\right)^{\frac{p}{2}} d \sigma(z) \rightarrow 0
$$

Proof. Let Area $\left(B_{h}(i, u(r))\right)=\pi \sinh ^{2} u(r)$ be the Euclidean area of $B_{h}(i, u(r))$. Then it follows that

$$
\begin{aligned}
E(r) & \leq \operatorname{Area}\left(B_{h}(i, u(r))\right) \sup _{z \in B_{h}(i, u(r))} \rho_{\mathbf{H}}(z)^{2-p}\left(\int_{\mathbf{H}(r)}|K(\bar{z}, \zeta)|^{2} d \sigma(\zeta)\right)^{\frac{p}{2}} \\
& <2^{p-2} \pi \sinh ^{2} u(r)(\log r)^{p-2}\left(\int_{\mathbf{H}(r)} \frac{d \sigma(\zeta)}{\min _{z \in B_{h}(i, u(r))}|\zeta-\bar{z}|^{4}}\right)^{\frac{p}{2}} \\
& =2^{p-2} \pi\left(\frac{\log r}{\sinh u(r)}\right)^{p-2}\left(\sinh ^{2} u(r) \int_{\mathbf{H}(r)} \frac{d \sigma(\zeta)}{\min _{z \in B_{h}(i, u(r))}|\zeta-\bar{z}|^{4}}\right)^{\frac{p}{2}} .
\end{aligned}
$$

By the proof of Lemma 3.2 in [20], $\log r / \sinh u(r) \rightarrow 1$ and

$$
\sinh ^{2} u(r) \int_{\mathbf{H}(r)} \frac{d \sigma(\zeta)}{\min _{z \in B_{h}(i, u(r))}|\zeta-\bar{z}|^{4}} \rightarrow 0
$$

as $r \rightarrow \infty$. Therefore the lemma holds. 


\section{Main theorems}

This section deals with the proof of the main theorems. We first prove the smoothness of $h_{\mathrm{WP}}^{p}$, which is based on [2,3].

Theorem 5.1. Let $\Gamma$ be a Fuchsian group with Lehner's condition and $p \geq 2$. If $p$ is an even number, then the $p$-Weil-Petersson metric $h_{\mathrm{WP}}^{p}$ is $C^{\infty}$-smooth on $T^{1,0} T^{p}(\Gamma) \backslash\{0\}$, otherwise $h_{\mathrm{WP}}^{p}$ is $C^{\lfloor p\rfloor-1}$-smooth on $T^{1,0} T^{p}(\Gamma) \backslash\{0\}$. Here $\lfloor\cdot\rfloor$ is the floor function.

Proof. The definition of $h_{\mathrm{WP}}^{p}$ depends essentially on right translation maps, whose action is nothing but replacing each point $\tau$ in $T^{p}(\Gamma)$ with the base point in $T^{p}\left(\Gamma^{\tau}\right)$. Then it is sufficient to consider the behavior of $h_{\mathrm{WP}}^{p}$ on the chart $U_{0}$ at the base point $T^{p}(\Gamma)$. We identify $U_{0}$ with $V_{0} \subset \operatorname{HB}^{p}(\mathbf{H}, \Gamma)$. Note that the holomorphic tangent vector space at each point in $V_{0}$ can be regarded as $\operatorname{HB}^{p}(\mathbf{H}, \Gamma)$.

Let us first consider the Gâteaux $z$-derivative of $h_{\mathrm{WP}}^{p}$. We define $h_{\mu}(\xi+t \kappa)$ as $h_{\mathrm{WP}}^{p}(\xi+t \kappa, \mu)$ for $\xi \in V_{0}, \mu, \kappa \in \operatorname{HB}^{p}(\mathbf{H}, \Gamma) \backslash\{0\}$, and $t \in \mathbf{C}$ with $\xi+t \kappa \in V_{0}$. We first deal with the case of $\xi=0$. Set $\mu(t)=L^{t \kappa}[\mu], \tilde{\mu}(t)=H[\mu(t)], \kappa(t)=L^{t \kappa}[\kappa]$. Then we have

$$
\begin{aligned}
h_{\mu}(t \kappa)^{p} & =\|\tilde{\mu}(t)\|_{p}^{p}=\int_{N(t)}|\tilde{\mu}(t)(z)|^{p} \rho_{\mathbf{H}}(z)^{2} d \sigma(z) \\
& =\int_{N}\left|A_{t}(z)\right|^{p}\left|K_{t}(z, \bar{z})\right|^{1-p}\left(1-|t \kappa(z)|^{2}\right) d \sigma(z),
\end{aligned}
$$

where $f^{t}=f^{t \kappa}, N(t)=f^{t}(N), K_{t}=K_{t \kappa}$ and

$$
A_{t}(z)=\tilde{\mu}(t)\left(f^{t}(z){\overline{\partial f^{t}(z)}}^{2} \rho_{\mathbf{H}}\left(f^{t}(z)\right)^{2}=\frac{3}{\pi} \int_{\mathbf{H}} \mu(\zeta) K_{t}(\bar{z}, \zeta)^{2} d \sigma(\zeta) .\right.
$$

Then we obtain

$$
D_{G}^{\prime} h_{\mu}(t \kappa)[\kappa]=\frac{\partial h_{\mu}}{\partial t}(t \kappa)=\frac{1}{p} h_{\mathrm{WP}}^{p}(t \kappa, \mu)^{1-p}\left(I_{1}+I_{2}+I_{3}\right),
$$

where

$$
\begin{aligned}
I_{1} & =\int_{N}\left|A_{t}(z)\right|^{p}\left|K_{t}(z, \bar{z})\right|^{1-p} \frac{\partial}{\partial t}\left(1-|t \kappa(z)|^{2}\right) d \sigma(z) \\
& \left.=-\bar{t} \int_{N}\left|A_{t}(z)\right|^{p}\left|K_{t}(z, \bar{z})\right|^{1-p}|\kappa(z)|^{2}\right) d \sigma(z), \\
I_{2} & =\int_{N}\left|A_{t}(z)\right|^{p} \frac{\partial}{\partial t}\left|K_{t}(z, \bar{z})\right|^{1-p}\left(1-|t \kappa(z)|^{2}\right) d \sigma(z) \\
& =(1-p) \int_{N}\left|A_{t}(z)\right|^{p} K_{t}(z, \bar{z})\left|K_{t}(z, \bar{z})\right|^{-p-1} \frac{\partial}{\partial t} K_{t}(z, \bar{z})\left(1-|t \kappa(z)|^{2}\right) d \sigma(z), \\
I_{3} & =\int_{N} \frac{\partial}{\partial t}\left|A_{t}(z)\right|^{p}\left|K_{t}(z, \bar{z})\right|^{1-p}\left(1-|t \kappa(z)|^{2}\right) d \sigma(z) \\
& =\frac{p}{2} \int_{N}\left(\overline{A_{t}(z)} \frac{\partial}{\partial t} A_{t}(z)+A_{t}(z) \frac{\partial}{\partial t} \overline{A_{t}(z)}\right)\left|A_{t}(z)\right|^{p-2}\left|K_{t}(z, \bar{z})\right|^{1-p}\left(1-|t \kappa(z)|^{2}\right) d \sigma(z) .
\end{aligned}
$$

Note that $h_{\mathrm{WP}}^{p}(t \kappa, \mu) \neq 0$ by Condition (1) in Definition 3.1. We have to show the commutativity between the signs of differentiation and integration in the above computation. It is sufficient to prove that $I_{1}, I_{2}, I_{3}$ converge absolutely and uniformly with respect to $t$ in an open neighborhood of 0 . 
We first consider $I_{1}$. Let $D_{1}(t, r)=N(t) \cap B_{h}(i, u(r))$ and $N(t, r)=N(t) \backslash D_{1}(t, r)$ for $r>e$ as Lemma 4.3. We note that the family $\left\{D_{1}(t, r) \mid r>e\right\}$ is an exhaustion of $N(t)$. There exists a constant $\delta_{1}>0$ such that $\left(|t|^{2}+|t|\right)|\kappa(z)|^{2}<1$ for $|t|<\delta_{1}$ and $z \in \mathbf{H}$. This implies that

$$
\int_{\left(f^{t}\right)^{-1}(N(t, r))}\left|A_{t}(z)\right|^{p}\left|K_{t}(z, \bar{z})\right|^{1-p}|t||\kappa(z)|^{2} d \sigma(z) \leq\left\|\left.\tilde{\mu}(t)\right|_{N(t, r)}\right\|_{p}^{p} .
$$

Since $H$ is a bounded linear operator, we have

$$
\left\|\left.\tilde{\mu}(t)\right|_{N(t, r)}\right\|_{p}^{p} \leq\|H\|^{p}\left\|\left.\mu(t)\right|_{N(t, r)}\right\|_{p}^{p} .
$$

By estimation (3.8) in [20], $\left\|\left.\mu(t)\right|_{N(t, r)}\right\|_{p}$ converges to 0 uniformly with respect to $t$ as $r \rightarrow 0$. Hence $I_{1}$ converges absolutely and uniformly with respect to $t$.

Next, we deal with $I_{2}$. By Lemmas 4.1 and 4.2 , for every $\varepsilon>0$, there exists a constant $0<\delta_{2}<1$ such that $\rho_{\mathbf{H}}(z)^{2}<(\varepsilon+1)\left|K_{t}(z, \bar{z})\right|$ and

$$
\left|\frac{\partial}{\partial t} K_{t}(z, \bar{z})\right|=\left|D^{\prime} F_{z}(t \kappa)[\kappa]\right|<\varepsilon\|\kappa\|_{\infty} \rho_{\mathbf{H}}(z)^{2}
$$

for $|t|<\delta_{2}$ and $z \in \mathbf{H}$. These inequalities imply that

$$
\begin{aligned}
\int_{\left(f^{t}\right)^{-1}(N(t, r))} & \left|A_{t}(z)\right|^{p}\left|K_{t}(z, \bar{z})\right|^{-p}\left|\frac{\partial}{\partial t} K_{t}(z, \bar{z})\right|\left(1-|t \kappa(z)|^{2}\right) d \sigma(z) \\
& <\varepsilon(\varepsilon+1)\|\kappa\|_{\infty} \int_{\left(f^{t}\right)^{-1}(N(t, r))}\left|A_{t}(z)\right|^{p}\left|K_{t}(z, \bar{z})\right|^{1-p}\left(1-|t \kappa(z)|^{2}\right) d \sigma(z) \\
& =\varepsilon(\varepsilon+1)\|\kappa\|_{\infty}\left\|\left.\tilde{\mu}(t)\right|_{N(t, r)}\right\|_{p}^{p} .
\end{aligned}
$$

Therefore $I_{2}$ also converges absolutely and uniformly with respect to $t$ as the case of $I_{1}$.

The last part is to check the convergence of $I_{3}$. Since

$$
\begin{aligned}
\frac{\partial}{\partial t} K_{t}(\bar{z}, \zeta) & =-\frac{1}{\pi} \int_{\mathbf{H}} \frac{\overline{\partial f^{t}(z)} \partial f^{t}(\zeta)}{\left(w-\overline{f^{t}(z)}\right)^{2}\left(w-f^{t}(\zeta)\right)^{2}} \kappa(t)(w) d \sigma(w), \\
\frac{\partial}{\partial t} A_{t}(z) & =\frac{6}{\pi} \int_{\mathbf{H}} \mu(\zeta) K_{t}(\bar{z}, \zeta) \frac{\partial}{\partial t} K_{t}(\bar{z}, \zeta) d \sigma(\zeta), \\
\frac{\partial}{\partial t} \overline{A_{t}(z)} & =\frac{6}{\pi} \int_{\mathbf{H}} \overline{\mu(\zeta)} K_{t}(z, \bar{\zeta}) \frac{\partial}{\partial t} K_{t}(z, \bar{\zeta}) d \sigma(\zeta),
\end{aligned}
$$

we have

$$
\begin{aligned}
I_{3}=-\frac{3 p}{\pi^{2}}\left\{\int_{N(t) \times \mathbf{H}}\right. & \overline{\tilde{\mu}(t)(z)}|\tilde{\mu}(t)(z)|^{p-2} \mu(t)(\zeta) T_{\kappa(t)}(\bar{z}, \zeta) K(\bar{z}, \zeta) d \sigma(z, \zeta) \\
& \left.+\int_{N(t) \times \mathbf{H}} \tilde{\mu}(t)(z)|\tilde{\mu}(t)(z)|^{p-2} \overline{\mu(t)(\zeta)} T_{\kappa(t)}(z, \bar{\zeta}) K(z, \bar{\zeta}) d \sigma(z, \zeta)\right\}
\end{aligned}
$$

where

$$
\int_{N(t) \times \mathbf{H}} \cdots d \sigma(z, \zeta)=\int_{N(t)}\left(\int_{\mathbf{H}} \cdots d \sigma(\zeta)\right) d \sigma(z)
$$

and

$$
T_{\kappa(t)}(\bar{z}, \zeta)=\int_{\mathbf{H}} \kappa(t)(w) K(w, \bar{z}) K(w, \zeta) d \sigma(w) .
$$

It is sufficient to show the absolute and uniform convergence of the first integral. Let $D_{2}(r)=\mathbf{H} \cap B_{e}(0, r)$ for $r>e$. Then $\left\{D_{1}(t, r) \times D_{2}(r) \mid r>e\right\}$ is an exhaustion of 
$N(t) \times \mathbf{H}$. The complement of $D_{1}(t, r) \times D_{2}(r)$ in $N(t) \times \mathbf{H}$ has the disjoint union decomposition

$$
(N(t) \times \mathbf{H}) \backslash\left(D_{1}(t, r) \times D_{2}(r)\right)=(N(t, r) \times \mathbf{H}) \sqcup\left(D_{1}(t, r) \times \mathbf{H}(r)\right),
$$

where $\mathbf{H}(r)=\mathbf{H} \backslash D_{2}(r)$. It is sufficient to show that two integrals

$$
\begin{aligned}
& I_{3}^{1}(t, r)=\int_{N(t, r) \times \mathbf{H}}\left|\tilde{\mu}(t)(z)^{p-1} \mu(t)(\zeta) T_{\kappa(t)}(\bar{z}, \zeta) K(\bar{z}, \zeta)\right| d \sigma(z, \zeta), \\
& I_{3}^{2}(t, r)=\int_{D_{1}(t, r) \times \mathbf{H}(r)}\left|\tilde{\mu}(t)(z)^{p-1} \mu(t)(\zeta) T_{\kappa(t)}(\bar{z}, \zeta) K(\bar{z}, \zeta)\right| d \sigma(z, \zeta)
\end{aligned}
$$

converge to 0 uniformly with respect to $t$ as $r \rightarrow 0$. It follows from the Schwarz inequality that

$$
I_{3}^{1}(t, r) \leq \int_{N(t, r)}|\tilde{\mu}(t)(z)|^{p-1}\|\mu(t) K(\bar{z}, \cdot)\|_{2}\left\|T_{\kappa(t)}(\bar{z}, \cdot)\right\|_{2} d \sigma(z),
$$

where

$$
\begin{aligned}
\|\mu(t) K(\bar{z}, \cdot)\|_{2} & =\left(\int_{\mathbf{H}}|\mu(t)(\zeta) K(\bar{z}, \zeta)|^{2} d \sigma(\zeta)\right)^{\frac{1}{2}}, \\
\left\|T_{\kappa(t)}(\bar{z}, \cdot)\right\|_{2} & =\left(\int_{\mathbf{H}}\left|T_{\kappa(t)}(\bar{z}, \zeta)\right|^{2} d \sigma(\zeta)\right)^{\frac{1}{2}} .
\end{aligned}
$$

By Hölder's inequality, we have

$$
\begin{aligned}
\|\mu(t) K(\bar{z}, \cdot)\|_{2}^{p} & \leq\left(\int_{\mathbf{H}}|\mu(t)(\zeta)|^{p}|K(\bar{z}, \zeta)|^{2} d \sigma(\zeta)\right)\left(\int_{\mathbf{H}}|K(\bar{z}, \zeta)|^{2} d \sigma(\zeta)\right)^{\frac{p}{2}-1} \\
& =\pi^{\frac{p}{2}-1} \rho_{\mathbf{H}}(z)^{p-2} \int_{\mathbf{H}}|\mu(t)(\zeta)|^{p}|K(\bar{z}, \zeta)|^{2} d \sigma(\zeta) .
\end{aligned}
$$

We apply the isometry property on the Hilbert transform. Then we obtain

$$
\begin{aligned}
\left\|T_{\kappa(t)}(\bar{z}, \cdot)\right\|_{2}^{2} & =\pi^{2} \int_{\mathbf{H}}|K(w, \bar{z}) \kappa(t)(w)|^{2} d \sigma(w) \\
& \leq \pi^{2}\|\kappa(t)\|_{\infty}^{2} \int_{\mathbf{H}}|K(w, \bar{z})|^{2} d \sigma(w)=\pi^{3}\|\kappa(t)\|_{\infty}^{2} \rho_{\mathbf{H}}(z)^{2} .
\end{aligned}
$$

It follows from inequalities (5.2), (5.3) and Hölder's inequality that

$$
\begin{aligned}
I_{3}^{1}(t, r) & \leq \pi^{\frac{3}{2}}\|\kappa(t)\|_{\infty} \int_{N(t, r)}|\tilde{\mu}(t)(z)|^{p-1} \rho_{\mathbf{H}}(z)\|\mu(t) K(\bar{z}, \cdot)\|_{2} d \sigma(z) \\
& \leq \pi^{\frac{3}{2}}\|\kappa(t)\|_{\infty}\left\|\left.\tilde{\mu}(t)\right|_{N(t, r)}\right\|_{p}^{p-1}\left(\int_{N(t, r)} \rho_{\mathbf{H}}(z)^{2-p}\|\mu(t) K(\bar{z}, \cdot)\|_{2}^{p} d \sigma(z)\right)^{\frac{1}{p}} \\
& \leq \pi^{2-\frac{1}{p}}\|\kappa(t)\|_{\infty}\left\|\left.\tilde{\mu}(t)\right|_{N(t, r)}\right\|_{p}^{p-1}\left(\int_{N(t) \times \mathbf{H}}|\mu(t)(\zeta)|^{p}|K(\bar{z}, \zeta)|^{2} d \sigma(z, \zeta)\right)^{\frac{1}{p}}
\end{aligned}
$$


Let us transform the integral in the last line of this inequality. By the invariance of $\mu(t)$ and $K(\bar{z}, \zeta)$ on the action of $\Gamma^{t \kappa}$, we have

$$
\begin{aligned}
& \int_{N(t) \times \mathbf{H}}|\mu(t)(\zeta)|^{p}|K(\bar{z}, \zeta)|^{2} d \sigma(z, \zeta) \\
& =\sum_{\gamma \in \Gamma^{t \kappa}} \int_{N(t) \times \gamma(N(t))}|\mu(t)(\zeta)|^{p}|K(\bar{z}, \zeta)|^{2} d \sigma(z, \zeta) \\
& =\sum_{\gamma \in \Gamma^{t \kappa}} \int_{\gamma^{-1}(N(t)) \times N(t)}|\mu(t)(\gamma(\zeta))|^{p}|K(\overline{\gamma(z)}, \gamma(\zeta))|^{2}\left|\gamma^{\prime}(z)\right|^{2}\left|\gamma^{\prime}(\zeta)\right|^{2} d \sigma(z, \zeta) \\
& =\int_{N}|\mu(t)(\zeta)|^{p}\left(\int_{\mathbf{H}}|K(\bar{z}, \zeta)|^{2} d \sigma(z)\right) d \sigma(\zeta)=\pi\|\mu(t)\|_{p}^{p} .
\end{aligned}
$$

Therefore, we obtain

$$
I_{3}^{1}(t, r) \leq \pi^{2}\|\kappa(t)\|_{\infty}\|\mu(t)\|_{p}\left\|\left.\tilde{\mu}(t)\right|_{N(t, r)}\right\|_{p}^{p-1} .
$$

Since $\|\kappa(t)\|_{\infty}$ and $\|\mu(t)\|_{p}$ is uniformly bounded on a neighborhood at $t=0, I_{3}^{1}(t, r)$ converges to 0 uniformly with respect to $t$ as $r \rightarrow 0$.

By similar estimation, we have

$$
\begin{aligned}
I_{3}^{2}(t, r) & \leq \pi^{\frac{3}{2}}\|\kappa(t)\|_{\infty} \int_{D_{1}(t, r)}|\tilde{\mu}(t)(z)|^{p-1} \rho_{\mathbf{H}}(z)\left\|\left.\mu(t) K(\bar{z}, \cdot)\right|_{\mathbf{H}(r)}\right\|_{2} d \sigma(z) \\
& \leq \pi^{\frac{3}{2}}\|\kappa(t)\|_{\infty}\|\mu(t)\|_{\infty}\|\tilde{\mu}(t)\|_{p}^{p-1} E(r)^{\frac{1}{p}} .
\end{aligned}
$$

This inequality and Lemma 4.3 imply the uniform convergence of $I_{3}^{2}(t, r)$ with respect to $t$.

Therefore the computation of formula (5.1) is justified. It follows from the definition of $h_{\mathrm{WP}}^{p}$ that

$$
D_{G}^{\prime \prime} h_{\mu}(t \kappa)[\kappa]=\frac{\partial h_{\mu}}{\partial \bar{t}}(t \kappa)=\overline{\left(\frac{\partial \overline{h_{\mu}}}{\partial t}(t \kappa)\right)}=\overline{\left(\frac{\partial h_{\mu}}{\partial t}(t \kappa)\right)}=\overline{D_{G}^{\prime} h_{\mu}(t \kappa)[\kappa]} .
$$

Then $h_{\mu}$ has a $\bar{z}$-derivative at $t$. Substituting $t=0$ for $D_{G}^{\prime} h_{\mu}(t \kappa)[\kappa]$, we have

$$
\begin{aligned}
D_{G}^{\prime} h_{\mu}(0)[\kappa]= & -\frac{3}{\pi^{2}}\|\mu\|_{p}^{1-p}\left\{\int_{N \times \mathbf{H}} \overline{\mu(z)}|\mu(z)|^{p-2} \mu(\zeta) T_{\kappa}(\bar{z}, \zeta) K(\bar{z}, \zeta) d \sigma(z, \zeta)\right. \\
& \left.+\int_{N \times \mathbf{H}} \mu(z)|\mu(z)|^{p-2} \overline{\mu(\zeta)} T_{\kappa}(z, \bar{\zeta}) K(z, \bar{\zeta}) d \sigma(z, \zeta)\right\} \\
= & -\frac{3}{\pi^{2}}\|\mu\|_{p}^{1-p} \int_{N \times \mathbf{H}} \overline{\mu(z)} \mu(\zeta) \\
& \cdot\left(|\mu(z)|^{p-2}+|\mu(\zeta)|^{p-2}\right) T_{\kappa}(\bar{z}, \zeta) K(\bar{z}, \zeta) d \sigma(z, \zeta) .
\end{aligned}
$$

The last expression is obtained by changing the role between $z$ and $\zeta$ in the second integral in the middle expression and by transforming its integral as formula (5.4). Note that $I_{2}=0$ because $\left.\frac{\partial}{\partial t} K_{t}(z, \bar{z})\right|_{t=0}=0$. As the above computation, we obtain

$$
\begin{aligned}
D_{G}^{\prime} h_{\mu}(\xi)[\kappa]= & -\frac{3}{\pi^{2}}\|\tilde{\mu}(\xi)\|_{p}^{1-p} \int_{N(\xi) \times \mathbf{H}}\left(\overline{\tilde{\mu}(\xi)(z)}|\tilde{\mu}(\xi)(z)|^{p-2} \mu(\xi)(\zeta)\right. \\
& \left.+\tilde{\mu}(\xi)(\zeta)|\tilde{\mu}(\xi)(\zeta)|^{p-2} \overline{\mu(\xi)(z)}\right) T_{\kappa(\xi)}(\bar{z}, \zeta) K(\bar{z}, \zeta) d \sigma(z, \zeta)
\end{aligned}
$$


for $\xi \in V_{0}$, where $\mu(\xi)=L^{\xi}[\mu], \tilde{\mu}(\xi)=H[\mu(\xi)]$ and $\kappa(\xi)=L^{\xi}[\kappa]$. This implies that $D_{G}^{\prime} h_{\mu}(\xi)$ is a complex linear map. Similarly to the estimation for $I_{3}^{1}(t, r)$, we have

$$
\left|D_{G}^{\prime} h_{\mu}(\xi)[\kappa]\right| \leq 6\|\mu(\xi)\|_{\infty}\|\kappa(\xi)\|_{p} \leq 6\|\mu(\xi)\|_{\infty}\left\|L^{\xi}\right\|\|\kappa\|_{p} .
$$

Hence $D_{G}^{\prime} h_{\mu}(\xi)$ is a bounded complex linear functional on $\operatorname{HB}^{p}(\mathbf{H}, \Gamma)$ and $h_{\mathrm{WP}}^{p}$ is Gâteaux differentiable on $V_{0}$.

Let us consider $n$ times differentiablity of $h_{\mathrm{WP}}^{p}$ at the base point for $n \geq 2$. This observation depends essentially on the smoothness of the function $P(z)=|z|^{p}$ on $\mathbf{C}$ for $p \geq 2$. In fact, $f(z)$ is $C^{\infty}$-smooth on $\mathbf{C}$ if $p$ is an even number, otherwise $P(z)$ is $C^{\lfloor p\rfloor-1}$-smooth on $\mathbf{C}$ because the $\lfloor p\rfloor$-th derivative of $P(z)$ has a singularity at $z=0$. The integrands in the $n$-th derivative of $\|\tilde{\mu}(t)\|_{p}^{p}$ with respect to $t$ always contain one of the following terms:

(1) The derivative of $\left(1-|t \kappa(z)|^{2}\right)$ (cf. $\left.I_{1}\right)$;

(2) The $k$-th derivative of $\left|K_{t}(z, \bar{z})\right|^{1-p}$ (cf. $\left.I_{2}\right)$;

(3) The $k$-th derivative of $\left|A_{t}(z)\right|^{p}$ (cf. $I_{3}$ ).

Here $1 \leq k \leq n$ and $z \in \mathbf{H}$. Case (1) makes no problem since $|t \kappa(z)|^{2}$ is $C^{\infty}$-smooth on some sufficiently small neighborhood $W$ at 0 . In case $(2)$, since $K_{t}(z, \bar{z}) \neq 0$ for every $z \in \mathbf{H}$ and every $t \in W,\left|K_{t}(z, \bar{z})\right|^{1-p}$ is $C^{\infty}$-smooth on $W$. We have to check the higher order differentiablity of case (3). For $z \in \mathbf{H}$, the function $A_{t}(z)$ is $C^{\infty}$ smooth on $W$. By this fact and the smoothness of $P(z)$, which was remarked above, $\left|A_{t}(z)\right|^{p}$ is $C^{\infty}$-smooth on $W$ when $p$ is an even number, otherwise $\left|A_{t}(z)\right|^{p}$ is $C^{\lfloor p\rfloor-1}$ smooth on $W$ generally. We can prove the commutativity between differentiation and integration by repeating or combining the methods used in the estimation of $I_{m}$ $(m=1,2,3)$. From these results, our claim is satisfied completely.

Let us prove the main theorem of this paper.

Theorem 5.2. Let $\Gamma$ be a Fuchsian group with Lehner's condition and $p \geq 2$. Then $h_{\mathrm{WP}}^{p}$ is strongly pseudoconvex on $T^{p}(\Gamma)$.

Proof. It suffices to show the strong pseudoconvexity at the base point. By a simple computation similar to that in the proof of Theorem 5.1, we obtain

$$
\begin{aligned}
& \left.\frac{\partial^{2}}{\partial t \partial \bar{t}} h_{\mathrm{WP}}^{p}(0, \mu+t \kappa)^{2}\right|_{t=0} \\
& =\|\mu\|_{p}^{2-2 p}\left\{\frac{p}{2}\|\mu\|_{p}^{p} \int_{N}|\mu(z)|^{p-2}|\kappa(z)|^{2} \rho_{\mathbf{H}}(z)^{2} d \sigma(z)\right. \\
& \left.\quad-\left.\left.\left(\frac{p}{2}-1\right)\left|\int_{N}\right| \mu(z)\right|^{p-2} \overline{\mu(z)} \kappa(z) \rho_{\mathbf{H}}(z)^{2} d \sigma(z)\right|^{2}\right\}
\end{aligned}
$$

for every $\mu, \kappa \in \operatorname{HB}^{p}(\mathbf{H}, \Gamma) \backslash\{0\}$. Note that the commutativity between the signs of differentiation and integration clearly follows. By the Schwarz inequality, we have

$$
\left.\left.\left|\int_{N}\right| \mu(z)\right|^{p-2} \overline{\mu(z)} \kappa(z) \rho_{\mathbf{H}}(z)^{2} d \sigma(z)\right|^{2} \leq\|\mu\|_{p}^{p} \int_{N}|\mu(z)|^{p-2}|\kappa(z)|^{2} \rho_{\mathbf{H}}(z)^{2} d \sigma(z) .
$$

Hence it follows that

$$
\left.\frac{\partial^{2}}{\partial t \partial \bar{t}} h_{\mathrm{WP}}^{p}(0, \mu+t \kappa)^{2}\right|_{t=0} \geq\|\mu\|_{p}^{2-p} \int_{N}|\mu(z)|^{p-2}|\kappa(z)|^{2} \rho_{\mathbf{H}}(z)^{2} d \sigma(z) .
$$

This implies that $h_{\mathrm{WP}}^{p}$ is strongly pseudoconvex at 0 since $\mu, \kappa \neq 0$. 


\section{References}

[1] Abate, M., and G. Patrizio: Finsler metrics - a global approach. - Lecture Notes in Math. 1591, Springer-Verlag, Berlin, 1994.

[2] Ahlfors, L. V.: Curvature properties of Teichmüller's space. - J. Anal. Math. 9, 1961, 161176.

[3] Ahlfors, L. V.: Some remarks on Teichmüller space of Riemann surfaces. - Ann. of Math. (2) $74,1961,171-191$.

[4] Ahlfors, L. V., and L. Bers: Riemann's mapping theorem for variable metrics. - Ann. of Math. (2) 72, 1960, 385-404.

[5] CuI, G.: Integrably asymptotic affine homeomorphisms of the circle and Teichmüller spaces. Sci. China Ser. A 43, 2000, 267-279.

[6] Hui, G.: Integrable Teichmüller spaces. - Sci. China Ser. A 43, 2000, 47-58.

[7] Lehner, J.: On the boundedness of integrable automorphic forms. - Illinois J. Math. 18, 1974, $575-584$.

[8] Matsuzaki, K.: Rigidity of groups of circle diffeomorphisms and Teichmüller spaces. arXiv:1607.06316.

[9] Nag, S., and A. Verjovsky: Diff $\left(\mathbf{S}^{1}\right)$ and the Teichmüller space. - Comm. Math. Phys. 130, 1990, 123-138.

[10] Niebur, D., and M. Sheingorn: Characterization of Fuchsian groups whose integrable forms are bounded. - Ann. of Math. (2) 106, 1977, 239-258.

[11] Radnell, D., E. Schippers, and W. Staubach: A Hilbert manifold structure on the WeilPetersson class Teichmüller space of bordered Riemann surfaces. - Commun. Contemp. Math. 17:1550016, 2015, 1-42.

[12] Radnell, D., E. Schippers, and W. Staubach: Weil-Petersson class non-overlapping mappings into a Riemann surface. - Commun. Contemp. Math. 18:1550060, 2016, 1-21.

[13] Radnell, D., E. Schippers, and W. Staubach: Convergence of the Weil-Petersson metric on the Teichmüller space of bordered Riemann surfaces. - Commun. Contemp. Math. 19:1650025, 2017, 1-39.

[14] Rajeswara RaO, K. W.: On the boundedness of $p$-integrable automorphic forms. - Proc. Amer. Math. Soc. 44, 1974, 278-282.

[15] Takhtajan, L. A., and L.-P. TeO: Weil-Petersson metric on the universal Teichmüller space. - Mem. Amer. Math. Soc. 183, Amer. Math. Soc., 2006.

[16] TANG, S.: Some characterizations of the integrable Teichmüller space. - Sci. China Math. 56, 2013, 541-551.

[17] Wolpert, S.: The Fenchel-Nielsen deformation. - Ann. of Math. (2) 115, 1982, 501-528.

[18] Wolpert, S.: On the Weil-Petersson geometry of the moduli space of curves. - Amer. J. Math. 107, 1985, 969-997.

[19] Yanagishita, M.: Introduction of a complex structure on the $p$-integrable Teichmüller space. - Ann. Acad. Sci. Fenn. Math. 39, 2014, 947-971.

[20] YanAGishita, M.: Kählerity and negativity of Weil-Petersson metric on square integrable Teichmüller space. - J. Geom. Anal. 27, 2017, 1995-2017. 